\title{
RETRIEVAL OF “RECOVERY IVC FILTER(TM)" AFTER 383 DAYS
}

\author{
Jiri Kozak, Martin Köcher, Rohit Philip Thomas, Marie Cerna, Stanislav Burval
}

Department of Radiology, University Hospital, Faculty of Medicine and Dentistry, Palacky University, I.P.Pavlova 6, 775 20

Olomouc, Czech Republic.

jiri.kozak@fnol.cz

Received: March 16, 2009; Accepted: December 2, 2009

Key words: Deep vein thrombosis/Pulmonary embolism/Recovery IVC filter ${ }^{(T M)} /$ Anti thrombin III deficiency/Recovery Cone removal system

Aim: Prolonged temporary implantation of retrievable vena cava filter is little discussed in literature. The aim of

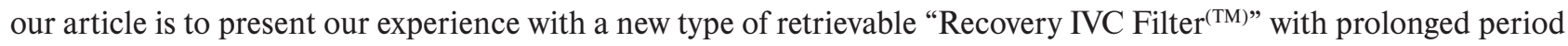
of placement in the inferior vena cava (IVC).

Materials and methods: "Recovery IVC Filter ${ }^{(\mathrm{TM})}$ " was implanted in another department for a 27 year old patient with congenital deficiency of anti thrombin III with deep vein thrombosis (DVT) complicated by pulmonary embolism (PE). After 383 days from implantation of vena cava filter, the patient was indicated for removal of the filter from the right jugular vein access.

Results: Filter was removed with the help of a special extraction system without significant technical complications in our department. On control phlebography after extraction of cava filter, there were no signs of injury to the wall of inferior vena cava, extravasation or stenosis.

Conclusion: Removal of "Recovery IVC Filter (TM)" after long term temporary implantation is possible and safe.

\section{INTRODUCTION}

DVT of the lower extremities complicated by PE is relatively a frequent cause of death in hospitalized patients and a very relevant complication in pregnancy. The risk of DVT and associated PE is especially frequent in patients with derangement of coagulation parameters. In the Czech Republic incidence of DVT is reported as $0.3 \%$ and incidence of $\mathrm{PE}$ as $0.06 \%$.

The basic treatment of DVT or PE is medical treatment in the form of anticoagulation (Heparin, Low molecular weight heparin (LMWH) and Warfarin). When the treatment is not effective as in case of repeated PE or progressing DVT in spite of adequate anticoagulation therapy or when there are contraindications to anticoagulation therapy or in case of complications associated with anticoagulation therapy, the next method of choice for prophylaxis of PE is IVC filter.

Three types of IVC filters are being used in clinical practice - temporary, permanent and retrievable filters recently. While the implantation of temporary filters is associated with high risk of occurrence of early local complications and implantation of permanent filter is associated with high risk of occurrence of late complications, the new type of retrievable filter minimizes the risk associated with temporary and permanent filters while retaining the advantage of prolonged implantation with the possibility of late removal in high risk patients who require long-term prophylaxis of PE. Prolonged temporary implantation of retrievable cava filter is less discussed in the literature. The aim of our article is to present our experience with the new type of retrievable "Recovery IVC Filter $^{(\mathrm{TM})}$ " (Bard Peripheral Vascular, Tempe, AZ, USA) with prolonged placement in IVC for a patient with congenital deficiency of anti thrombin III and DVT progressing to PE shortly after delivery.

\section{CASE REPORT}

A 27 year old young woman (Czech citizen living in USA) was presented at the emergency unit of a hospital in Chicago (Saint Joseph Hospital, Chicago, USA) with approximately one week of edema and pain of the left lower extremity. Doppler ultrasound examination revealed thrombosis of the popliteal vein, superficial femoral vein and common femoral vein. The woman was in her tenth week of gestation. According to the family history, her father was treated twice in the past for DVT complicated by PE with detected anti thrombin III deficiency. Therefore the patient was subjected to complete blood examination where reduced levels of anti thrombin III (74\%, normal range $83-122 \%$ ) and positive lupus anticoagulants were detected. The remaining hypercoagulability factors (Protein C, Protein S, Factor V Leiden, Prothrombin gene mutation and homocystein) were normal. During the period of gestation, patient was treated with low molecular weight heparin - Lovenox (Sanofi-Aventis U.S.). Treatment was continued with Lovenox in doses $60 \mathrm{mg}$ twice daily from the 3 rd week after delivery with gradual modification to warfarin - Coumadin (Bristol-Myers Squibb Company).

Six weeks after delivery, the patient was again presented to the emergency (Saint Joseph Hospital, Chicago, USA) with edema of the left lower extremity and chest pain associated with 2-3 days persisting hemoptysis. 
Computed Tomography (CT) angiography was performed, from which PE was diagnosed. Owing to the high risk of fatal PE and since she was already treated by adequate medical treatment (Lovenox and Coumadin) and was in a hypercoagulable state, patient was indicated for implantation of "Recovery IVC Filter ${ }^{(\mathrm{TM})}$ ". The procedure was carried out without problems at the hospital in Chicago under ultrasound and fluoroscopic control from a right sided femoral access. "Recovery IVC Filter ${ }^{(\mathrm{TM})}$ " was composed of 12 0.014-inch nitinol wires. It had six flexible legs that anchor the device to the caval wall and six arms, creating a dual-level filtration system.

The patient was later admitted to our department (Department of Radiology, University Hospital, Olomouc, Czech Republic) for the removal of Recovery IVC filter after 383 days. Before the procedure, warfarin (Orion Corporation, Espoo, Finland) was changed for low molecular weight heparin-Fraxiparin (Glaxo Wellcome Production, Notre Dame de Bondeville, France). The procedure was carried out from right sided jugular access with the use of a special extraction system ("Recovery Cone removal system", C.R. Bard) where the jugular vein was punctured under ultrasound control and with the help of guide wire, a special $10 \mathrm{~F}$ sheath was introduced.

Phlebography was then performed using a $4 \mathrm{~F}$ pigtail catheter which was introduced into the IVC below cava filter (Fig. 1, 2, 3). The IVC was patent with no signs of filling defects in the filter. The filter was placed correctly in the longitudinal axis of the IVC below the origin of the renal arteries. There were no signs of penetration of filter struts to the wall of the vein. Subsequently the 4F pigtail was removed using guide wire and only the $10 \mathrm{~F}$ sheath and guide wire were left in the lumen of the IVC. The sheath for the easiest removal of filter was aligned as near as possible to the centre of the filter itself.

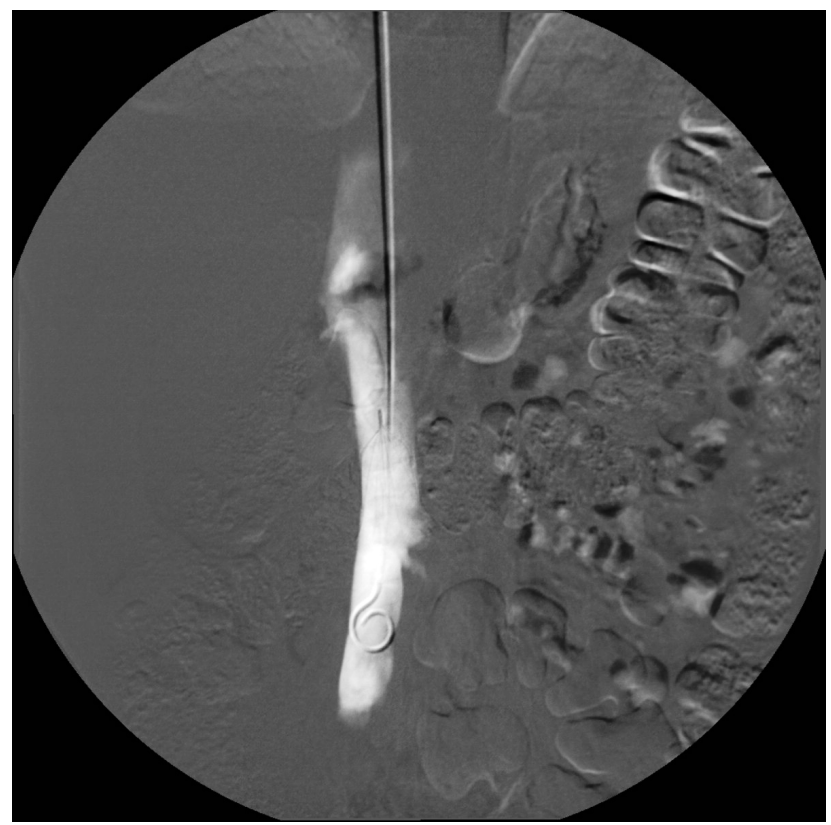

The extraction system consisting of 9 metal struts covered by urethane foil was introduced into the IVC through the $10 \mathrm{~F}$ sheath using the guide wire. Using this special extraction system, the filter was safely caught and subsequently pulled to the sheath and retrieved. On control phlebography, no injury of the wall of IVC or no signs of extravasation were found and IVC was fully patent (Fig. 4, 5). The filter was not damaged and no presence of thrombus was detected. The procedure was finished with removal of the sheath from the jugular vein and short compression of the puncture site.

\section{DISCUSSION}

Long-term placement of cava filter constitutes a safe alternative for the prophylaxis of PE in high risk patients especially patients in thrombophilic state, patients with polytrauma, immobile patients with brain and spine injuries and in patients with DVT complicated by $\mathrm{PE}^{1-5}$

For long-term vena cava filtration, permanent filters are commonly used, however long-term placement of these filters is associated with a high degree of complication. Similarly temporary utilization of filter is associated with possible early complication. Therefore a new type of filter was created - removable cava filter which is constructed like a permanent filter, but the difference is the possibility of removal after several weeks.

Retrievable filters have become more widely accepted because they offer the advantages of short-term PE prophylaxis, while avoiding immediate risks of temporary filters and the potential long-term risks of permanent filters $^{3}$. Placement of retrievable cava filter for the prophylaxis of PE constitutes the main option for patients who require temporary filtration ${ }^{6}$.

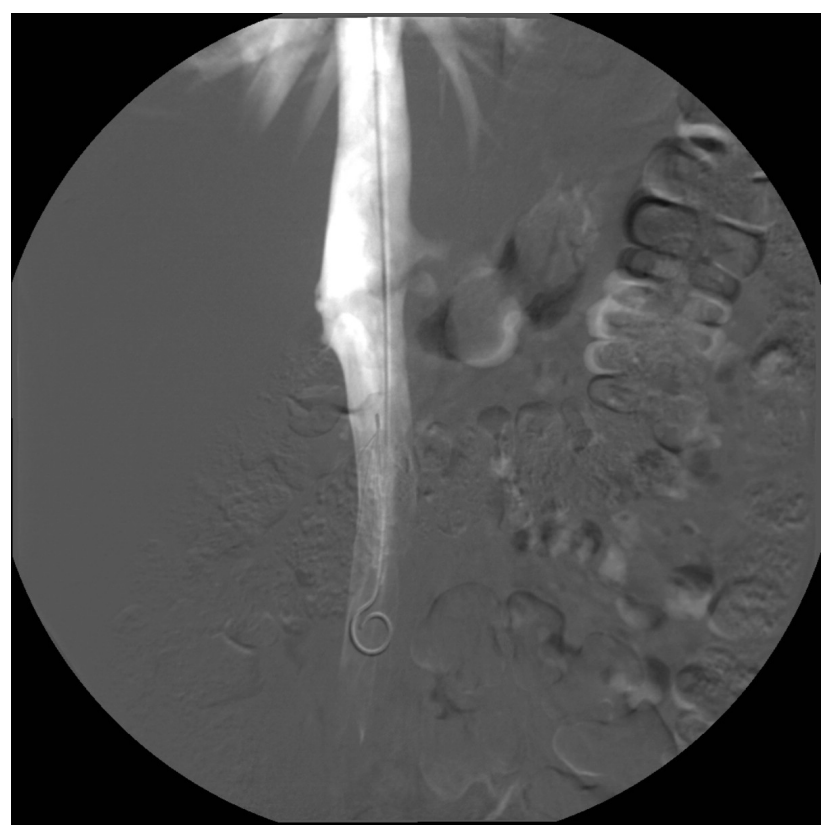

Fig. 1-2. Cavogram before the extraction of filter from inferior vena cava confirming correct position of fiter in the long axis, smooth walls of inferior vena cava and no presence of thrombus in the filter and free patent inferior vena cava. 
Generally it is known that the period of implantation of the filter with safe removal is decided by the manufacturer and is up to 14 days ("Opt Ease Filter" has approved period of implantation of 23 days in USA). These days in the market, a few types of filters are commonly used, which are approved to be removable or permanent and can be utilized for prolonged temporary implantation with late removal ${ }^{1,5}$. These are "Opt Ease Filter" (Cordis, Warren, NJ), "Günther Tulip Filter" (Cook, Bloomington, IN, USA) and "Recovery IVC Filter ${ }^{(\mathrm{TM}) \text { " (Bard Peripheral }}$ Vascular, Tempe, AZ, USA), up-grade version "Recovery G2 IVC Filter"(Bard). In Europe, "ALN Filter” (ALN Implants Chirurgicaux, Ghisonaccia, France) are used like removable filters. Recently in the market a new type of removable filter - "Celect Filter" (Cook, Bloomington, IN, USA) has been introduced which is similar to the "Recovery IVC Filter ${ }^{(\mathrm{TM})}$ " and is used for prolonged temporary implantation. Although long-term placement of the cava filter is advantageous from the point of PE prophylaxis, the placement is also associated with possible late complications ${ }^{1,3}$. Among these, the main late complications are cava thrombosis with repeated PE, filter migration $4,6,7$, filter fracture and cava penetration with or without perforation ${ }^{6,8}$. Filter fractures are less frequent and are reported less than $1 \%$ in the literature ${ }^{6}$.

A few studies and case reports have been published about prolonged temporary implantation with use of "Recovery IVC Filter ${ }^{(\mathrm{TM})}$ "1-5,8,9.

The first report about the use of "Recovery IVC

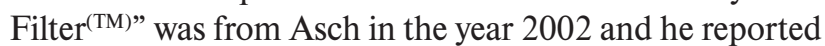
an average period of implantation 53 days (5-134 days) in which out of 32 implanted filters, 26 were successfully retrieved. Trapped thrombus was seen in 7 patients and cranial migration of filter in one patient ${ }^{4}$.

In another study which comprised 106 cava filter implanted patients with average duration of implantation

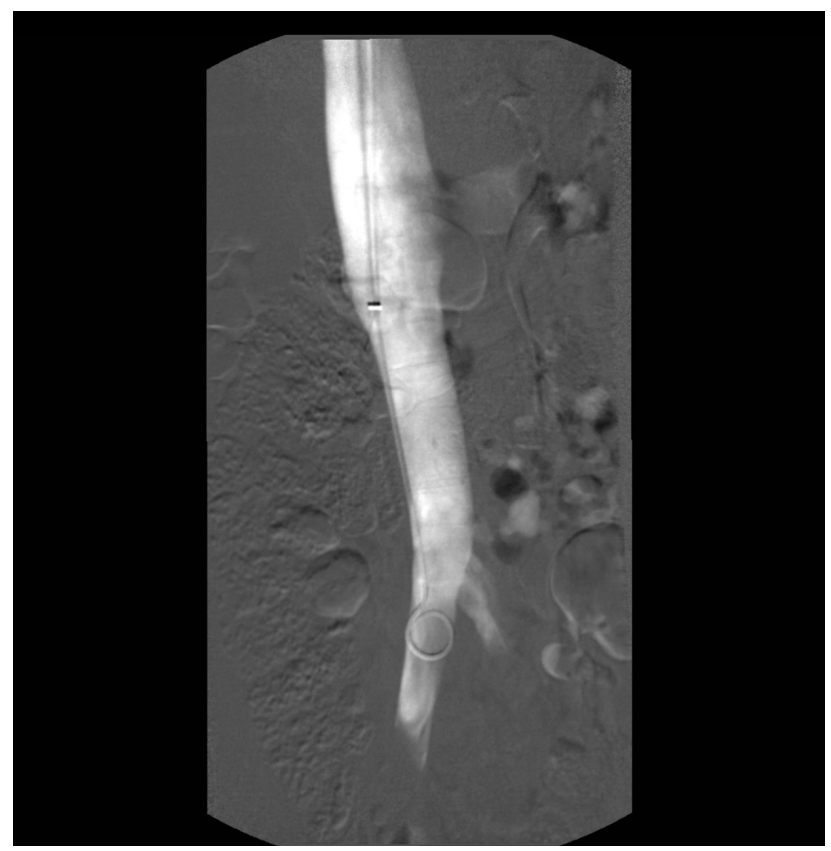

of 150 days, it was reported that removal of filter was successful in 14 out of 15 attempts (93\%) and the authors report symptomatic PE as a complication and it was only $2.8 \%$ (ref.) ${ }^{1}$.

In another study which comprised 13 patients with implantation period from 181 to 419 days, the authors reported successful retrieval in $100 \%$. All filters were retrieved successfully without difficulties. Two of 13 filters had a tilt of more than $10^{\circ}$, but no filter was embedded into the IVC wall and no filter migration or thrombus within the filter was observed ${ }^{3}$.

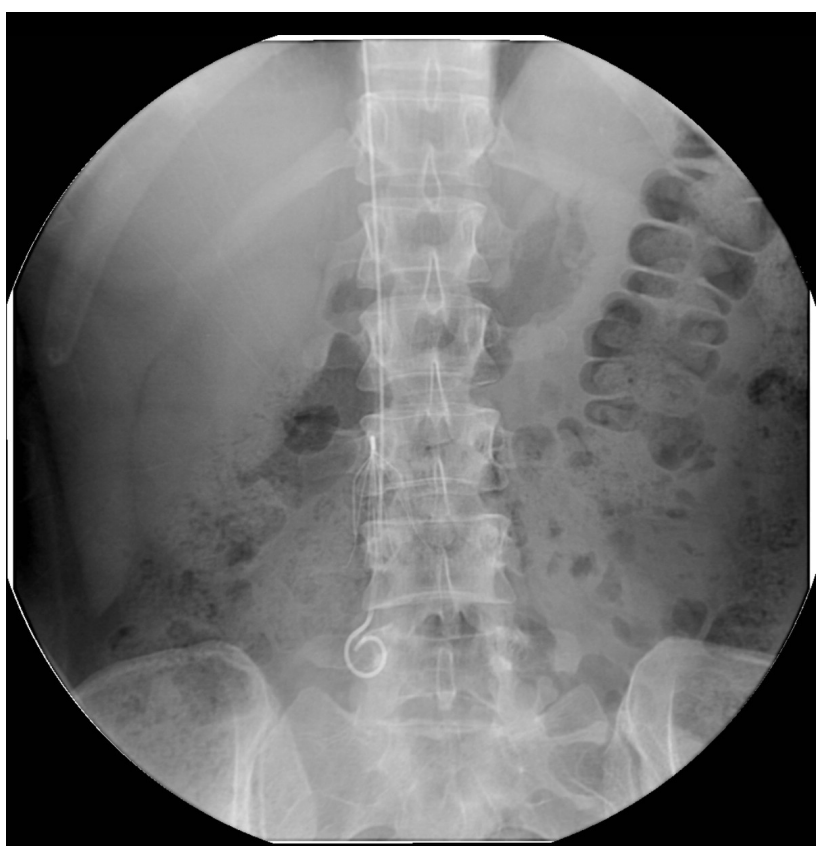

Fig. 3. Native picture with introduced pigtail in inferior vena cava placed below the cava filter which is without any injury of the filter skeleton.

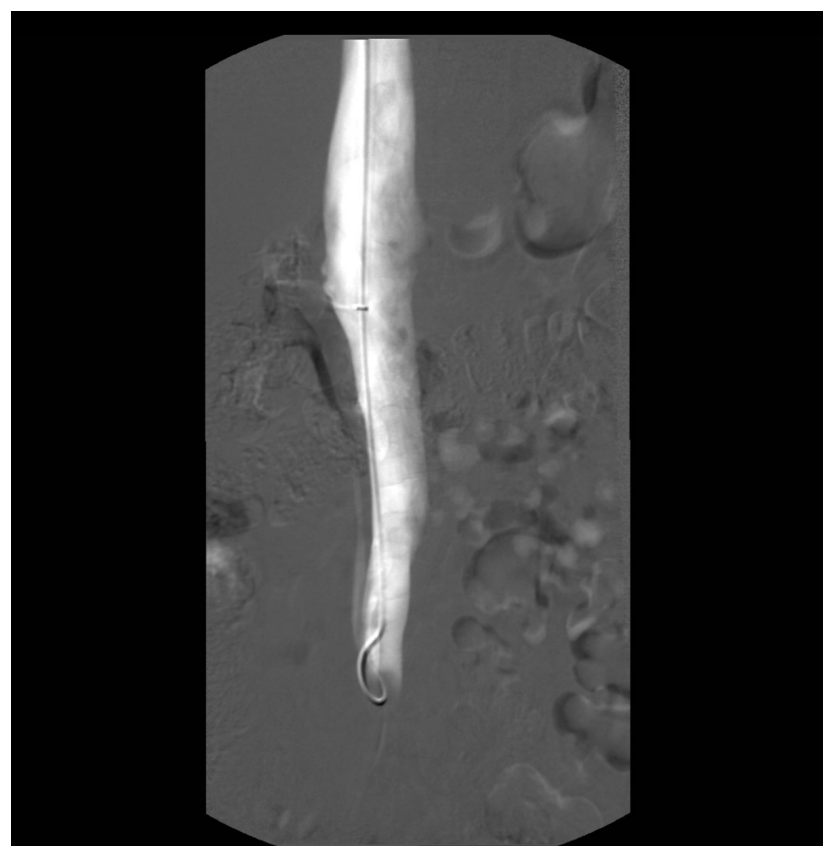

Fig. 4-5. Control cavogram after removal of filter where patent inferior vena cava is seen with smooth walls and without signs of extravasation. 
In one recent study on 96 patients with an average implantation period of 117 days (24-426), the authors reported 11 attempts to remove the filter with successful removal in nine patients (82\%). Follow-up abdominal CT (40 patients) at a mean of 80 days (1-513) showed penetration of the IVC by the filter arms in 11, other complications were fracture of filter components in 3 patients, and fracture of filter legs with penetration to pancreas in one patient ${ }^{8}$.

From these data, it is seen that the percentage of removed filter after prolonged temporary implantation is not very high and if successful, the percentage of removal is relatively high because of the rarer complications. We may assume that long term placement of cava filter as such does not have high risk of late removal.

However it is necessary to take into real consideration that the maximum average period of implantation of filter with its subsequent removal does not exceed 150 days $^{1}$ in these studies and only in some cases the filter was removed after more than a year. Even in recently published studies, the maximum period for safe extraction of filter after prolonged implantation is not defined definitely.

However when we take into consideration results from prospective randomized studies which have been reported, the implantation of cava filter longer than 24 months in patients with anticoagulation therapy significantly increases thrombosis compared to patients who received only anticoagulation therapy ${ }^{10}$. From this it is evident that temporary implantation of removable cava filter longer than 2 years loses its effect and on the contrary increases the risk of complication especially risk of repeatable PE, risk of migration, penetration and perforation of filters.

\section{CONCLUSION}

Our experiences with retrievable "Recovery IVC Filter ${ }^{(\mathrm{TM})}$ " after prolonged temporary implantation albeit it is one, is good. To our knowledge, this is the first report of extraction of such a filter with the special extraction system in Europe after temporary implantation as long as 383 days. We removed the filter without any problems after 383 days of implantation and without any of the complications mentioned above. Removal of this type of filter after a period of implantation more than 12 months is possible.

\section{ABBREVIATIONS}

CT - Computed tomography; DVT - Deep vein thrombosis; IVC - Inferior vena cava; PE - Pulmonary embolism

\section{REFERENCES}

1. Grande WJ, Trerotola SO, Reilly PM, et al. Experience with the recovery filter as a retrievable inferior vena cava filter. J Vasc Interv Radiol 2005;16:1189-1193.

2. Lipman JC. Removal of vena caval filter at 224 days. South Med J. 2005 May;98(5):556-8.

3. Binkert CHA, Sasadeusz K, Stavropoulos W. Retrievability of recovery vena cava filter after dwell times longer than 180 days. J Vasc Radiol 2006;17:299-302.

4. Asch MR, FRCPC. Initial experience in humans with a new retrievable inferior vena cava filter. Radiology 2002;225:835-844.

5. Bérczi V, Bottomley JR, Thomas SM, Taneja S, Gaines PA, Cleveland TJ. Long-Term retrievability of IVC filters: should we abandon permanent devices? Cardiovasc Intervent Radiol 2007 Sept-Oct;30:820-827.

6. Ganguli S, Tham JC, Dmitry JR. Fracture and migration of suprarenal inferior vena cava filter in a pregnant patient. J Vasc Interv Radiol 2006;17:1707-1711.

7. Izutani H, Lalude O, Gill IS, Biblo IA. Migration of an inferior vena cava to the right ventricle and literature review. Can J Cardiol 2004; 20:233-235.

8. Kalva SP, Athanasoulis CA, Fan CM, Curvelo M, Geller SC, Greenfield AJ, Waltman AC, Wicky S. "Recovery“ vena cava filter: experience in 96 patients. Cardiovasc Intervent Radiol 2006 Jul-Aug;29(4):559-564.

9. Hagspiel KD, Lejny DA, Alladin M, et al. Difficult retrieval of a recovery IVC filter. J Vasc Interv Radiol 2004;15:645-647.

10. Decousus H, Leizorovicz A, Parent F, et al. Five-year follow up of prophylactic vena cava filters in the prevention of pulmonary embolism in patients with proximal deep-vein thrombosis. Prevention du Risque d'Embolie Pulmonaire par Interruption Cave Study Group. N Engl J Med 1998;338:409-415. 